\title{
Closure of the Open Abdomen: a Practical Approach
}

\author{
Yoram Klein ${ }^{1}$
}

Published online: 11 October 2016

(C) Springer International Publishing AG 2016

\begin{abstract}
Purpose of Review This review focusing on the closure of the open abdomen which is one of the most complicated challenges of the modern critical care surgeon.

Recent Findings Three phases of open abdomen can be defined. The first is from the primary damage control operation until all necessary intra-abdominal interventions were concluded. The second is from the end of the first period until closure of the wound. If by the end of the second phase the fascia could not be closed, the skin only coverage is mandated. The third phase is between the skin coverage to the definitive abdominal wall reconstruction. During the first two phases, the abdomen should be covered by a temporary closure technique that should minimize the open abdomen-related complications and facilitate delayed primary closure of the abdomen.

Summary Modern temporary closure uses negative pressure therapy in combination with medial traction on the fascial edge. This approach gives the best chance for early delayed primary closure and minimal complication rate.
\end{abstract}

Keywords Damage control laparotomy $\cdot$ Abdominal wound closure techniques $\cdot$ Negative pressure wound therapy .

Enteroatmospheric fistula

This article is part of the Topical Collection on Abdominal Compartment Syndrome in Trauma

Yoram Klein

yoram.klein@sheba.health.gov.il

1 Trauma Division, Department of Surgery B, Sheba Medical Center, Tel Hashomer, Ramat Gan, Israel

\section{Introduction}

The concept of leaving the abdomen open after damage control laparotomy has revolutionized the management of trauma and non-trauma-related abdominal surgical catastrophes. There are several reasons for using this dramatic measure. The most common indication is to treat or prevent abdominal compartment syndrome (ACS). The second common indication is the need for an early re-laparotomy in cases such as packing for bleeding, heavily contaminated abdomen, or ischemic bowel. While the indications for open abdomen and the related intra-abdominal procedures have not changed in recent decades, the closure technique has evolved significantly. Both technological advancement and better physiological understanding are the basis for this evolution.

The open abdomen (OA) can be divided into three phases. The first is the period in which repeated intra-abdominal procedures, such as unpacking, debridement, or bowel viability evaluation, are still needed. The second is the period in which no other intra-abdominal intervention is needed but the abdominal wall cannot be approximated yet, due to tissue edema. If after the second phase, the fascia cannot be closed, a skin only coverage of the wound (either primarily or with partially thickness skin graft) is mandated. The time between the skin coverage until the definitive abdominal wall reconstruction is the third phase. Every phase has its own characteristics, risks, and complications. In this article, we will delineate the fundamental elements of the closure of the OA in a practical view.

\section{Pathophysiology of OA}

The OA carries several systemic and local problems. Fluid, protein, electrolytes, and heat loss are the most important systemic problems, while adhesions and bowel perforations are 
the most serious local complications. The patient in the first phase usually suffers from severe metabolic derangement secondary to cardiovascular instability, hemorrhage, sepsis, or systemic inflammation that resulted from the primary insult. In most circumstances, the first damage control operation should be abbreviated, and the patient should be promptly transferred to the intensive care unit for optimal vital systems support. A repeated operation for unpacking, regaining bowel continuity, evaluation for organ viability, establishment of enteric diversion, or debridement of necrotic or infected tissue is usually needed after no more than $48 \mathrm{~h}$. In cases of severe intra-abdominal infection, such as infected necrotizing pancreatitis, debridement every $48 \mathrm{~h}$ should be repeated several more times. During this time, excessive amount of cytokinerich peritoneal fluid is secreted into the peritoneal cavity. Absorption of this fluid to the systemic circulation is taught to carry malicious remote effects and metabolic derangement [1]. By the end of the first phase, definitive fascial closure should be attempted. If not possible due to bowel and retroperitoneal edema, the abdomen should be kept open. Usually, this period is characterized by better hemodynamic stability and improving metabolic state. Tissue edema should subside considerably to allow tension-free abdominal wall closure. On the other hand, a natural wound healing process takes place, starting from the injurious event that causes adhesions between bowel loops, and between the viscera to the anterior and lateral parietal peritoneum. At the same time, there is a continuous process of contraction of the wound edges. If the abdomen is not closed within a period of 8-10 days, the end result of this process is a frozen visceral sac that is fixed to the peritoneal gutters and lateralization of the fascial edges that cause widened wound, loss of domain, and eventually inability to primarily close the fascia $[2,3]$.

Probably the most feared complication of the OA is bowel perforation. This is probably due to exposure of the bowel to the unfavorable conditions of the OA, such as dryness, mechanical shearing forces from dressing changes, or even relative movement of the bowel against the coverage sheath. Iatrogenic inadvertent serosal injury is also an important factor in the development of perforations. When the perforation is not covered by a well perfused viable tissue and it is exposed in the wound, there is an established enteroatmospheric fistula (EAF) - the ultimate nightmare of every surgeon who deals with an OA.

\section{Temporary Closure}

In the first two phases of OA, temporary closure (TC) is used in order to reduce the occurrence of OA-related complications. The TC goals include an atraumatic coverage of the exposed abdominal viscera with low adhesive potential to the bowel, preventing abdominal hypertension, minimizing fluid, electrolytes, proteins and heat loss, allowing easy re-entry to the abdomen, and improving the chance for delayed primary closure (DPC) of the abdominal wall $[4,5]$. The first TC of OA was described by Sir William Ogilvie during the Second World War [6]. He used light canvas, sterilized with Vaseline to bridge laparotomy wounds that could not be primarily closed. This technique has evolved into the famous "Bogota bag" in which a nylon sheath that is sutured to the fascia or skin edges [7]. This technique is a passive silo that accomplishes some of the goals of TC. Its main advantage is its simplicity, availability (any sterile empty IV solution bag can be used), quick application, and low cost. On the other hand, it has two main limitations. First, the high volume fluid secretions from the abdomen are difficult to control with this modality. Second, its mechanism of action does not promote DPC. The modern TC modalities are an active dressing that uses the negative pressure therapy (NPT) principles. The dressing has three basic components. The first is a fenestrated atraumatic transparent sheath that protects and covers the exposed viscera while allowing fluid to pass through the fenestrations. This layer, if located properly, all the way down to the gutters also serves as a barrier between the viscera and the parietal peritoneum. This may prevent the local adhesions and delay the unwanted fixation of the visceral sac, and by that prolongs the time until DPC will not be possible. The second component is a wound filler that should be used as an absorptive layer that transmits the negative pressure to the abdominal cavity and also keeps a moisture environment to the wound. The negative pressure also creates some medial traction on the wound edges, which counteract the natural retraction, to a certain degree. The third layer is an adhesive drape that occludes the abdominal cavity and allows the negative pressure to be effectively built within the abdomen. The negative pressure is created by a tube, located beneath the collusive drape that is connected to either a pump or the wall suction. It is the most effective way to drain and collect the secretions from the abdomen. Except for dramatically improve the nursing care, in comparison to the passive dressing, there is also an active removal of cytokine-rich fluid from the abdominal cavity. Correlation between the cytokine concentration in the peritoneal secretion, the level of systemic circulating cytokines, and end organ dysfunction has been described in few studies [8•]. The active removal of the abdominal fluid is believed to ameliorate its systemic remote effects $[9 \bullet \bullet, 10]$. Negative pressure is known to promote creation and proliferation of granulation tissue. This, together with the medial traction force that this technique imposes on the wound edges, promotes healing process, reducing the gap between the two wound edges and, in general, accelerates and improves the chance for DPC. The NPT-based TP can be either a commercial product or an improvised dressing. The most common commercial product is the ABthera (KCI, San Antonia, TX, USA). It constructed of a fenestrated nonadherent layer with encapsulated foam that is placed within 
the abdominal cavity, a foam that is placed over the covered wound surface, a drape, which is applied over the abdomen and a suction tubing that is placed over a hole cut in the drape to have direct contact with the foam. The negative pressure is produced by a special pump. The Barker's technique, also known as the poor man vac, is the most popular improvised NPT dressing. A sterile nylon cover is usually used as a protective barrier between the viscera and the wound filler, which can be either laparotomy pads or a gauze-based material. A large adhesive sheath such as steri-drape (3 M health care, St. Paul, MN, USA) covers the dressing above one or two tubes that are connected to the wall suction. This dressing is cheap and simple to use. Delayed primary closure rate with this technique was reported to be $55 \%$, with only $4 \%$ EAF [11•]. One of the theoretical shortcoming of poor man vac, compare to the commercial devices, is the inability to control and accurately set up the level of the negative pressure. Anyhow, to date, no clinical evidence exists to support the importance of this feature. We use $-125 \mathrm{mmHg}$ according to the manufacture recommendations, but no specific level of negative pressure was shown to be superior in the setup of OA. Most comparative studies demonstrated inferior performance of the Barker's compare to the ABthera $[12,13 \bullet \bullet]$. Nevertheless, this technique is still widely used and acceptable. In recent years, there is a growing body of literature on direct peritoneal resuscitation (DPR), as an adjunct to the initial resuscitation after a damage control laparotomy in hemodynamic compromised patients [14•]. The only TC technique that can practically be used during DPR is the Barker's technique, due to the copious amount of fluids that is instilled into the abdominal cavity and should be retrieved. The limited sized canister of the commercial NPT devices prohibited effective drainage of the abdomen during DPR.

The occurrence of EAF in patients with OA was reported to range from 0 to $54.8 \%\left[15 \bullet^{\circ}\right]$. One of the main concerns regarding the use of NPT in OA, is that imposing negative pressure on the friable bowel of a septic hypoalbuminemic patient, might promote bowel perforation or increase the leak from an already existing fistula. Currently available evidence do not support this theory despite its mechanical logic $[16,17 \bullet \bullet]$. EAF occurrence rate with NPT was found to be $14.6 \%$, and as low as $5.7 \%$ if combine with medial traction technique [15••]. There is a consensus that the single most important intervention that may reduce the risk of EAF is to close the $\mathrm{OA}$ as soon as possible. The fact that the NPT correlates with the shortest time to closure of an OA is well established. More than that, current guidelines recommend the VAC as the modality of choice for the management of EAF [18].

During the second phase, regression of bowel and retroperitoneal edema is the key element for tension-free delayed primary closure. Physiological wound healing process that starts immediately after the first operation actually aggravates the local conditions for primary closure. While correct application of NPT principles to the OA will reduce adhesions and fixation of the visceral sac to the abdominal wall, its ability to contradict wound edges (especially fascia) retraction and lateralization is limited. Applying continuous medial traction to the fascial edges can prevent this process and promote gradual progressive approximation of the wound edges. Several solutions are available for this purpose. The first commercial device that used this concept was the Wittman patch тM (Starsurgical Inc. Burlington, WI); it comprised by two nonpermeable synthetic sheaths that are sutured to the facial edges and attached together with a Velcro that can gradually be tightened. Another commercial solution is the abdominal reapproximation anchor (ABRA, Canica, Almonte, Ontario, Canada) that uses elastic bands crossing through the full thickness of the abdominal wall, and then progressively tensioned to approximate the fascial edges. Small series reported delayed primary closure rate of up to $88 \%$ with this system [19]. A common and effective improvisation of this concept is suturing polypropylene mesh to each facial edge and suturing the two pieces of the mesh in the midline. The desired effect is achieved by tightening the midline suture every dressing change [20]. The latest studies support the combination of both medial traction with NPT-based TC as the techniques of choice that provides the best chance for DPC in the shortest time with minimal occurrence of complications [21••]. Most studies showed that the expected DPC rate with the combination of NPT and medial traction should be above $85 \%$.

In our department, the default is to use the Barker's dressing during the first phase for two main reasons. First, the DPR is our standard of care in most post damage control patients. Secondly, we doubt the cost-effective rational for changing the relative expensive commercial dressing every $48 \mathrm{~h}$. With the NPT dressing, we use a prolene mesh as a medial traction solution. In the second phase, we change the Barker's dressing to a commercial NPT dressing. Dressing changes are being done usually every 72-96 h, during which we evaluate the wound for a possible closure. If after 14-20 days the fascia cannot be closed, we attempt component separation procedure for DPC. If failed or thought to have low chance of success, the skin will be mobilized to cover the open wound. If this also cannot be achieved, the wound will be left to granulate with ongoing NPT, and partial thickness skin graft will eventually be used to cover the wound. Our departmental algorithm is summarized in Fig. 1.

\section{Timing of Definitive Closure}

"As long as the abdomen is open, you control it. Once closed it controls you." This old surgical say demonstrate one of the dilemmas regarding definitive closure of the OA, especially when the indication was infected abdomen. The postoperative period is a stressful time for every surgeon. The most troubling question is always the possibility of intra-abdominal complication such as an infection, anastomotic leak, bowel ischemia, 
Fig. 1 Suggested algorithm for the closure of open abdomen. $O A$ open abdomen, $B D$ Barker's dressing, $M T$ medial traction, $V A C$ commercial negative pressure device

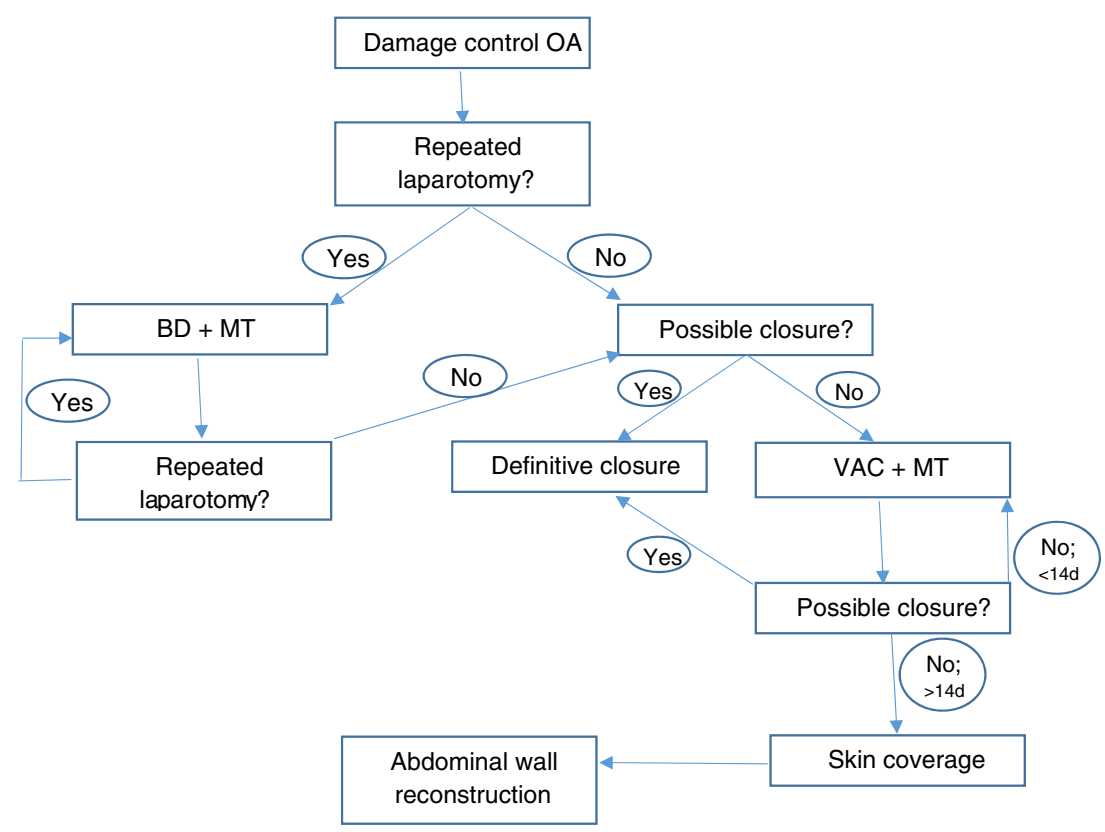

or bleeding. While the patient's abdomen is open and reexplored repeatedly during the first phase-this question is answered easily. When the abdomen is primarily closed or left to granulate, we lose the window to the abdominal cavity. The surgeon must recognize and overcome this psychological obstacle and remember that as, mentioned before, early closure is the most important intervention to reduce OA-related complications. These complications were shown to be more common after 2 weeks with an OA [22].

On the other hand, in a paraphrase on Einstein's famous words, every abdomen should be closed as early as possible, but not earlier. The advantages of closing the abdomen should be weighed against the possibility of repeated ACS, recurrent intra-abdominal infection, or the risk of abdominal wall ischemia secondary to tension.

\section{Definitive Closure}

In most patients, definitive closure can be achieved during the first or second phase. The first priority is for delayed primary closure. If tension prohibits simple fascia approximation, other methods can be successful, despite earlier recommendations that did not support component separation during the acute phase of OA [23]. Recent studies reported successful closure in up to $75 \%$ [24•]. If tension-free approximation cannot be achieved, bridging the remaining gap was suggested. The use synthetic mesh for abdominal closure after the first or second phase should be discouraged, due to the high rate of late complications, especially infected mesh [25]. Some recent small series reported promising results with the use of biological mesh. Early results were excellent, but the occurrence of late herniation is still unknown for lack of longterm follow-up [26-28]. Few methods for using myocutaneous advancement or free flaps were reported, but none gained popularity due to their complexity and the natural reservation from inflicting extensive tissue damage to the recovering friable patient.

If primarily closure of the fascia cannot be achieved by the end of the second phase, the only possible solution is planned ventral hernia. The granulated abdominal wound should be covered with the skin. This is achieved by creating subcutaneous flaps on both sides of the wound and closing the released skin in the midline. If the gap is too large to allow this technique, the wound should be covered with spilt thickness skin graft. I prefer the former approach, because according to our experience, definitive abdominal wall reconstruction can be achieve sooner. In cases of skin grafting, the reconstruction should be delayed until complete separation of the skin graft from the underlying tissue is evident (positive pinch test), usually after at least 10-12 months. The third phase should be concluded with an abdominal wall reconstruction as soon as possible because the planned hernia will continue to grow and cause significant discomfort and self-body image disturbance to the patients. This might interfere with his rehabilitation process and delays his return to reproductive and satisfactory life.

\section{Conclusions}

In cases of open abdomen, the surgeon must promote early delayed primary closure while minimizing complications. The best strategy to achieve these goals is temporary coverage of the abdomen with negative pressure improvised of commercial dressing with the addition of continuous and progressive medial traction of the fascial edges. If after 2-3 weeks the 
fascia cannot be closed with either direct suturing or after component separation technique, the abdomen should be covered by the skin only (either primary after the skin release by creating subcutaneous flaps, or with skin graft). The abdominal wall should be definitely reconstructed after $8-12$ months, when the skin coverage is well separated from the visceral sac beneath it. Following these principles will give these complicated patients the best chance for recovering and enhance their return to normal life.

\section{Compliance with Ethical Standards}

Conflict of Interest Dr. Klein declares no conflicts of interest relevant to this manuscript.

Human and Animal Rights and Informed Consent This article does not contain any studies with human or animal subjects performed by any of the authors.

\section{References}

Papers of particular interest, published recently, have been highlighted as:

- Of importance

•• Of major importance

1. Vidal MG, Ruiz Weisser J, Gonzalez F, Toro MA, Loudet C, Balasini $\mathrm{C}$, et al. Incidence and clinical effects of intra-abdominal hypertension in critically ill patients. Crit Care Med. 2008;36: 1823-31.

2. Miller PR, Thompson JT, Faler BJ, Meredith JW, Chang MC. Late fascial closure in lieu of ventral hernia: the next step in open abdomen management. J Trauma. 2002;53:843-9.

3. Miller RS, Morris JA, Diaz JJ, Herring MB, May AK. Complications after 344 damage-control open celiotomies. J Trauma. 2005;59:1365-71.

4. De Waele JJ, Leppaniemi AK. Temporary abdominal closure techniques. Am Surg. 2011;77 Suppl 1:S46-50.

5. Burlew CC, Moore EE, Cuschieri J, Jurkovich GJ, Codner P, Nirula $\mathrm{R}$, et al. Who should we feed? A Western Trauma Association multi-institutional study of enteral nutrition in the open abdomen after injury. J Trauma Acute Care Surg. 2012;73:1380-7.

6. Ogilvie WH. The late complications of abdominal war-wounds. Lancet. 1940;2:253-6.

7. Fernandez L, Norwood S, Roettger R, Wilkins III HE. Temporary intravenous bag silo closure in severe abdominal trauma. J Trauma. 1996;40:258-60.

8. Kubiak BD, Albert SP, Gatto LA, Snyder KP, Maier KG, Vieau CJ, et al. Peritoneal negative pressure therapy prevents multiple organ injury in a chronic porcine sepsis and ischemia/reperfusion model. Shock. 2010;34:525-34. An animal model that demonstrate the importance of removal of cytokines from the peritoneal cavity.

9.• Cheatham ML, Demetriades D, Fabian TC, Kaplan MJ, Miles WS, Schreiber MA, et al. Prospective study examining clinical outcomes associated with a negative pressure wound therapy system and Barker's vacuum packing technique. World J Surg. 2013;37:
2018-30. A prospective study that prove correlation between active fluid removal and improved outcome with $\mathrm{OA}$.

10. Kirkpatrick AW, Roberts DJ, Faris PD, Ball CG, Kubes P, Tiruta C, et al. Active negative pressure peritoneal therapy after abbreviated laparotomy: the intraperitoneal vacuum randomized controlled trial. Ann Surg. 2015;262:38-46.

11. Barker DE, Kaufman HJ, Smith LA, Ciraulo DL, Richart CL, Burns RP. Vacuum pack technique of temporary abdominal closure: a 7year experience with 112 patients. J Trauma. 2000;48:201-6. Retrospective study that showed the efficacy of improvised negative pressure dressing.

12. Frazee RC, Abernathy SW, Jupiter DC, Hendricks JC, Davis M, Regner JL, et al. Are commercial negative pressure systems worth the cost in open abdomen management? J Am Coll Surg. 2013;216: 730-3.

13.• Roberts DJ, Zygun DA, Grendar J, Ball CG, Robertson HL, Ouellet JF, et al. Negative-pressure wound therapy for critically ill adults with open abdominal wounds: a systematic review. J Trauma Acute Care Surg. 2012;73:629-39. A systemic review that summarizes the comparison between improvised and commercial negative pressure open abdomen closure dressings.

14. Smith JW, Garrison RN, Matheson PJ, Franklin GA, Harbrecht BG, Richardson JD. Direct peritoneal resuscitation accelerates primary abdominal wall closure after damage control surgery. J Am Coll Surg. 2010;210:658-64. The potential benefit of direct peritoneal resuscitation.

15.• Atema JJ, Gans SL, Boermeester MA. Systematic review and metaanalysis of the open abdomen and temporary abdominal closure techniques in non-trauma patients. World J Surg. 2015;39:912-25. The most comprehensive study on outcome and complications of temporary abdominal closure.

16. Shaikh IA, Ballard-Wilson A, Yalamarthi S, Amin AI. Use of topical negative pressure in assisted abdominal closure does not lead to high incidence of enteric fistulae. Colorectal Dis. 2010;12:931-4.

17.• Carlson GL, Patrick H, Amin AI, McPherson G, Maclennan G, Afolabi E, et al. Management of the open abdomen: a national study of clinical outcome and safety of negative pressure wound therapy. Ann Surg. 2013;257:1154-9. Negative pressure doesn't increase the occurrence of intestinal fistula.

18. Terzi C, Egeli T, Canda AE, Arslan NC. Management of enteroatmospheric fistula. Int Wound J. 2014;11 Suppl 1:17-21.

19. Verdam FJ, Dolmans DE, Loos MJ, Raber MH, de Wit RJ, Charbon JA, et al. Delayed primary closure of the septic open abdomen with a dynamic closure system. World J Surg. 2011;35:2348-55.

20. Petersson U, Acosta S, Bjorck M. Vacuum-assisted wound closure and mesh-mediated fascial traction - a novel technique for late closure of the open abdomen. World J Surg. 2007;31:2133-7.

21.•Willms A, Gusgen C, Schaaf S, Bieler D, von Websky M, Schwab R. Management of the open abdomen using vacuum-assisted wound closure and mesh-mediated fascial traction. Langenbecks Arch Surg. 2015;400:91-9. The combination of negative pressure and medial traction offer the best outcome for OA temporary closure.

22. Acosta S, Bjarnason T, Petersson U, Palsson B, Wanhainen A, Svensson M, et al. Multicentre prospective study of fascial closure rate after open abdomen with vacuum and mesh-mediated fascial traction. Br J Surg. 2011;98:735-43.

23. Diaz Jr JJ, Dutton WD, Ott MM, Cullinane DC, Alouidor R, Armen $\mathrm{SB}$, et al. Eastern association for the surgery of trauma: a review of the management of the open abdomen - part 2 management of the open abdomen. J Trauma. 2011;71:502-12.

24. Rasilainen SK, Mentula PJ, Leppäniemi AK. Components separation technique is feasible for assisting delayed primary fascial closure of open abdomen. Scand J Surg. 2016;105:17-21. A small clinical series that shows that component separation is a safe technique in the closure of $\mathrm{OA}$. 
25. Fansler RF, Taheri P, Cullinane C, Sabates B, Flint LM. Polypropylene mesh closure of the complicated abdominal wound. Am J Surg. 1995;170:15-8.

26. Guy JS, Miller R, Morris JA, Diaz J, May A. Early one-stage closure in patients with abdominal compartment syndrome: fascial replacement with human acellular dermis and bipedicle flaps. Am Surg. 2003;12:1025-8.
27. Singh MK, Rocca JP, Rochon C, Facciuto ME, Sheiner PA, Rodriguez-Davalos MI. Open abdomen management with human acellular dermal matrix in liver transplant recipients. Transplant Proc. 2008;40:3541-4.

28. Chuo CB, Thomas SS. Absorbable mesh and topical negative pressure therapy for closure of abdominal dehiscence with exposed bowel. J Plast Reconstr Aesthet Surg. 2008;61(11):1378-81. 